\title{
Belphégor
}

Littérature populaire et culture médiatique

$17 \mid 2019$

Mutations des légitimités dans les productions culturelles contemporaines

\section{Le dilettante et l'érudit : du discours académique à la critique romantique du savoir (Stendhal, Nerval)}

Juliette Mascart and Rémy Arcemisbéhère

\section{(2) OpenEdition}

\section{Journals}

Electronic version

URL: https://journals.openedition.org/belphegor/1634

DOI: $10.4000 /$ belphegor.1634

ISSN: 1499-7185

Publisher

LPCM

Electronic reference

Juliette Mascart and Rémy Arcemisbéhère, "Le dilettante et l'érudit : du discours académique à la critique romantique du savoir (Stendhal, Nerval)", Belphégor [Online], 17 | 2019, Online since 18 April 2019, connection on 25 August 2021. URL: http://journals.openedition.org/belphegor/1634 ; DOl: https://doi.org/10.4000/belphegor.1634

This text was automatically generated on 25 August 2021.

\section{(i) $\$$

Belphégor est mis à disposition selon les termes de la Licence Creative Commons Attribution - Pas d'Utilisation Commerciale - Pas de Modification 4.0 International. 


\title{
Le dilettante et l'érudit : du discours académique à la critique romantique du savoir (Stendhal, Nerval)
}

\author{
Juliette Mascart and Rémy Arcemisbéhère
}

1 Au tournant du XVIII ${ }^{e}$ siècle, l'accélération de l'Histoire altère fortement le rapport aux autorités traditionnelles et aux savoirs établis. On assiste alors à la constitution d'un nouveau champ épistémique fondé sur la conscience de la relativité des choses et des points de vue. Serge Zenkine estime ainsi que, dans le sillage des bouleversements sociopolitiques provoqués par la Révolution, l'idée même de culture subit une redéfinition épistémologique : la culture romantique, hautement réflexive, rejette les autorités transcendantales (celle $\mathrm{du}$ savant et du critique, qui définissent respectivement le savoir et le goût) et expérimente des codes multiples, ce qui se manifeste notamment par l'empan inédit du relativisme (géographique, historique et littéraire). Le projet romantique cherche à réconcilier de telle ou telle manière cette contradiction de la conscience culturelle, "celle d'une richesse sémiotique et d'une pauvreté herméneutique $»^{1}$, soit celle de la prolifération du sens et du rejet des instances. Deux auteurs du début du XIX ${ }^{\mathrm{e}}$ siècle incarnent ce phénomène contemporain du développement d'une sensibilité romantique : Stendhal et Nerval se situent tous deux en marge des institutions académiques et marquent un jalon de l'histoire culturelle qu'une archéologie des formes de l'érudition des amateurs se doit de prendre en compte.

2 Stendhal offre un exemple original d'écriture journalistique : dans les comptes rendus des représentations du Théâtre-Royal italien qu'il adresse de 1824 à 1827 au Journal de Paris, il met en lumière un type d'époque : le dilettante, amateur passionné de musique qui fréquente assidument cette institution. Contemporaine de l'introduction des œuvres de Rossini en France, la notion de dilettante fédère une jeunesse acquise aux principes romantiques et mue par un désir de renouveau. Dans sa critique musicale, 
Stendhal déplace cette notion d'un type d'époque à une démarche critique qu'il revendique.

3 En aval de ce changement de paradigme, Nerval tente quant à lui de déjouer les pièges de cette opposition entre la figure du dilettante et celle de l'érudit. Dans ses textes critiques, il ne manque jamais de fustiger les autorités culturelles: les "pointus littéraires » et leur souci de perfection classique ${ }^{2}$ ou encore les savants orientalistes comme Volney, De Sacy ou Ampère. Les récits nervaliens n'en proposent pas moins de longs passages de digressions savantes : le Voyage en Orient constitue un véritable parcours à travers la bibliothèque qui multiplie références, citations, parodies et plagiats (plus ou moins assumés), invoquant pêle-mêle textes littéraires, scientifiques ou encore mystiques. L'auteur y confronte ainsi les impressions du voyageur et les cadres disciplinaires de l'époque. De la même façon, le narrateur des nouvelles Angélique et Sylvie met en scène une érudition singulière à l'égard du folklore régional, rejetant les cadres doctrinaires et empruntant au promeneur ses attitudes et sa démarche.

Le rapprochement de ces deux auteurs dont le positionnement et les démarches diffèrent sensiblement, se justifie par la singularité revendiquée de leur rapport au savoir. Tous deux accomplissent un pas de côté épistémologique dont procède, en premier lieu, une analyse critique des postures du savoir où s'affirment le rejet des arrêts de l'opinion publique et, plus fondamentalement, le constat inquiet de la prolifération des autorités du savoir. En réaction à un savoir institué dont l'imposture est révélée, émergent les figures du polémiste et du révolté : nos deux auteurs s'inscrivent dans une posture contestataire pour fonder, s'il se peut, un nouvel ordre du savoir. On ne saurait unifier la portée de ces discours, qui engagent des conceptions très différentes de la culture et du savoir. Néanmoins, malgré leurs divergences, ces deux paradigmes de la critique romantique du savoir mènent à un même constat : celui de la réversibilité de tout discours critique. Ayant parcouru les formes académiques du discours de savoir et tâché de le refonder en l'inscrivant dans la tension constitutive du champ de la critique, Stendhal et Nerval font l'expérience de son aporie.

\section{Deux observateurs critiques des discours de savoir}

\section{La fabrique de l'opinion : faux savants et faux savoir}

Faisant la chronique des représentations données au Théâtre-Royal italien, Stendhal rend compte de l'événement sans s'embarrasser de considérations théoriques, adoptant la posture de l'amateur éclairé ${ }^{3}$ " [Voyant] des raisons pour soutenir toutes les opinions $»^{4}$, il se méfie de celles qu'on assène avec l'autorité de la science et s'attache à dénoncer le faux savoir, notamment les idées reçues qui dominent l'opinion publique, en France plus qu'ailleurs. Celles-ci fleurissent dans un temps où la bataille romantique oppose les tenants de la tradition aux partisans de la nouveauté, et ce particulièrement dans le champ musical qui voit renaître la querelle des Bouffons, ou du moins une rivalité marquée entre musique française et musique italienne. Sous la Restauration en effet, la musique tient une place de choix dans la vie mondaine : c'est alors « un genre, une mode " que de "se montr[er] assidu[ ] aux représentations du Théâtre-Italien », d'où l'émergence de la figure du " dilettante ", " amateur passionné, dans les affections duquel rien ne saurait remplacer la musique $»^{5}$. Ce terme, dont l'engouement rossiniste 
explique en partie le succès, est contemporain quant à son usage en langue française de l'introduction de la musique italienne en France au milieu du XVIIIe siècle et de la querelle qui s'ensuivit entre les partisans de Rameau et ceux de Gluck. Cet arrière-plan historique est souvent exploité dans la presse du temps au profit d'un nationalisme de mauvais aloi ${ }^{6}$ que Stendhal n'a de cesse de dénoncer : en disciple de Mme de Staël, il croit hautement profitable à une tradition moribonde de se regénérer au contact d'inspirations étrangères. Plus généralement, il redoute l'influence des journaux sur le public parisien, qu'il mesure au désarroi des amateurs censés se prononcer sur le mérite d'une œuvre nouvelle : «Toutes les premières représentations sont froides au Théâtre-Italien ; ce n'est guère que le surlendemain, quand les journaux ont parlé, que les dilettanti se hasardent à être ivres de plaisirs, ou à chuter ${ }^{7}$. L'insistance avec laquelle Stendhal dénonce le conformisme de ses pareils signale un enjeu d'importance, que révèle un éclairage contextuel. Sous le ministère de Villèle (décembre 1821-janvier 1828), le rapport au savoir fait l'objet d'une lutte acharnée entre les ultra-royalistes alors au pouvoir, et l'opposition libérale: sous l'action de la droite monarchique, le système d'enseignement tend de plus en plus à contrôler les esprits tout en établissant un conformisme politique et religieux des plus stricts. Stendhal, qui appréhende le phénomène dans sa globalité, explique ailleurs que le conformisme est devenu une dimension essentielle de la vie sociale contemporaine. De sorte que le public, prompt à applaudir ce dont il reconnaît la perfection formelle, demeure interdit devant la nouveauté :

Le public de Louvois, une fois accoutumé à voir telle roulade sur tel mot [...], paraît toujours mécontent si un nouvel acteur sent différemment son rôle et transporte à un autre mot, peignant une autre nuance de caractère, tout ce que sa voix peut avoir de charme, tout ce que son jeu peut présenter de chaleur.

6 Comme le fait observer Stendhal, ce public subit le joug d'« un ennemi bien puissant, l'esprit de routine $" ~{ }^{9}: 1$ 'habitude lui est un dogme dont il doit se déprendre pour accéder à la jouissance esthétique puisque " la nouveauté, qui, dans tous les beaux-arts, est la source des plaisirs, en musique est la condition sine qua non $»^{10}$. Qu'attendre cependant d'un public timoré qui n'ose se prononcer d'après son ressenti et qui « couvre de bravos ce que les journaux lui ont désigné comme étant beau $»^{11}$ ? La peur du ridicule et l'esprit d'imitation que dénote une telle attitude participent de cette vanité, toute française, qui consacre le faux savoir ${ }^{12}$ sous le vernis duquel se profile le faux savant. C'est ici la figure du pédant que révoque Stendhal, parce qu'elle participe d'une démarche qui fausse, voire annihile le jugement esthétique. Le même grief est exprimé par Nerval moins de cinq ans plus tard dans un article de mai 1830 où il dénonce le dogmatisme des " pointus littéraires », tournant en ridicule leur souci de perfection. En miroir de ces pointus littéraires, Nerval se revendique d'une autre critique, attentive seulement aux manifestations de la beauté, au point de heurter délibérément le credo rationaliste des Classiques :

Qu'il y a loin d'une telle critique à celle qu'affectionne l'École nouvelle; critique large et féconde, au regard de laquelle il n'est point de défauts si considérables qu'une véritable beauté ne puisse faire oublier, mais aussi pour qui rien ne peut compenser le manque de beautés ${ }^{13}$.

7 Nerval dénonce en ces " pointus littéraires » une école insensible à la beauté, occultée par les postulats critiques et les assomptions esthétiques, et appelle à un rapport naïf et sensible aux œuvres. 


\section{De la relativité des savoirs à la prolifération des légitimités}

De même que Stendhal, Nerval remet donc en cause la légitimité des discours de savoir. Ce processus affecte également le discours sur l'Orient : Daniel Lançon a montré que les méthodes du poète Gérard de Nerval et du savant Jean-Jacques Ampère divergent fondamentalement : Nerval travaille en effet à détourner l'érudition de la tâche que l'orientalisme lui fixe.

Le poète Nerval envisage ainsi une écriture du sujet qui ne soit pas pré-écrite au nom du collectif [...]. Gérard de Nerval exhibe les écrits arabes sur les pyramides, affectant de renier en partie la recherche rationnelle sur les monuments antiques au profit de l'accueil de l'imagination arabe et de ses « savoirs » on ne peut plus hétérodoxes aux yeux de l'Occident. ${ }^{14}$

Cette prise de conscience de la relativité des savoirs qui s'effectue à travers la réhabilitation de sources alternatives prend un tour plus personnel au cours du Voyage : comme Stendhal, Nerval réhabilite la part de l'expérience dans les mécanismes de production du savoir. Pourtant, ses nombreux récits de voyage trahissent un rapport ambigu à l'égard de ce principe : refusant l'opposition traditionnelle entre le savoir des livres et le savoir de l'expérience, le narrateur du Voyage en Orient observe que l'expérience de la lecture, de l'art ou encore du rêve constituent des références tout à fait légitimes. Après avoir été déçu par la découverte de la ville de Constance, bien pâle au regard de ses lectures d'enfance, le narrateur observe que :

C'est une impression douloureuse, à mesure qu'on va plus loin, de perdre, ville à ville et pays à pays, tout ce bel univers qu'on s'est créé jeune, par les lectures, par les tableaux et par les rêves. ${ }^{15}$

Cette inquiétude est réversible, puisque dans la nouvelle Isis, Nerval exprime sa crainte de ruiner l'expérience du voyage par ses lectures : " peut-être faut-il craindre, en voyage, de gâter par des lectures faites d'avance, l'impression première des lieux célèbres ${ }^{16}$.

Après avoir adopté la posture romantique de rejet des anciennes autorités au profit de nouvelles et affirmé la préséance des sources arabes sur celle de la littérature occidentale, autorité du rêve et des lectures sur l'expérience, l'auteur des Chimères ne manque pas de lucidité à l'égard du nécessaire corollaire d'une telle attitude face au savoir : la prolifération des légitimités. Dans le Voyage en Orient, Nerval tâche moins en effet d'établir une nouvelle légitimité que d'en confronter plusieurs : le récit de la visite de l'île de Cythère dans la suite de son Voyage en Orient manifeste la quantité des savoirs encyclopédiques accumulés sur l'île d'amour : savoirs mythologique (Homère, Virgile, Pausanias, Orphée), littéraires (Nodier, Goethe), religieux et mystiques (Francisco Colonna) et même scientifiques (les mythologues Creuzer et Guingiault) ${ }^{17}$. Cet épisode fait donc le choix de mettre en scène la prolifération des discours sur Cythère sans pour autant indiquer lequel doit faire autorité : pourtant, sur cette île, le voyageur ne rencontrera que des ruines muettes, et cette désillusion est aussi l'image de l'expérience de la perte des fondements du savoir. Il ne reste plus alors à l'auteur que la possibilité de mettre en scène ad nauseam cette prolifération. 


\section{Révolte et polémique : l'amateur en quête de légitimité} de légitimité qu'ils dénoncent hautement et sur lequel ils fondent une parole contestataire. Si Stendhal use de la polémique pour contrer un discours hégémonique, Nerval endosse la posture du révolté contre le savoir académique, pour réhabiliter le savoir des réprouvés.

\section{Une entreprise paradoxale}

amener ses lecteurs à reconsidérer les opinions courantes, Stendhal les met en cause de façon percutante. Sans entrer dans une démarche savante par nature froide et desséchante et partant tout à fait impropre à traiter des beaux-arts, il joue de son statut d'auteur des Vies de Haydn, de Mozart et de Métastase, et de la Vie de Rossini ${ }^{18}$ pour faire valoir son point de vue. Par un habile décentrement appuyé sur des considérations historiques, il transpose les termes de la polémique, affirmant qu' « [e]n musique, il y a deux routes pour arriver au plaisir : le style de Haydn et le style de Cimarosa, la sublime harmonie ou la mélodie délicieuse ${ }^{19}$. Invoquer la tradition allemande en lieu et place de la musique française permet à Stendhal de retrancher du débat sa dimension patriotique. Ces deux traditions (française et allemande) affirment le primat de l'harmonie sur la mélodie, et relèvent ainsi de la musique dite savante qui se prête malaisément, d'après Stendhal, au théâtre lyrique : «il me semble que l'harmonie est souvent une scène déplacée au théâtre. Elle règne dans la symphonie, dans la musique d'église ; mais au théâtre, on préfère en général une jolie cantilène de Paesiello aux morceaux d'orchestre les plus compliqués de Spohr ou de Winter » ${ }^{20}$. D'une phrase à l'autre, ce qui s'énonce d'abord comme un avis personnel («il me semble») épouse ensuite la forme d'un avis général (retrait de la première personne opéré par le biais d'une personnification, usage du pronom indéfini), l'opinion singulière se muant ainsi en idée reçue. Averti du conformisme ambiant, Stendhal s'applique à estomper le caractère original de ses propos de sorte qu'ils semblent participer de la doxa et sont reçus comme tels par ses lecteurs.

Pour efficace qu'elle soit, cette stratégie énonciative n'est pas systématique: ayant vérifié par ailleurs la force de la polémique, Stendhal se plait à heurter de front les préjugés établis afin de contrer la doxa imposée par les tenants de l'ordre établi et de rétablir une altérité menacée par le discours hégémonique. Aussi s'en prend-il, dans le domaine musical, au préjugé selon lequel la justesse d'intonation est le premier critère de qualité. Il soutient en effet que «[c]hanter faux quelquefois n'est pas, quoi qu'en disent les ignorants, le plus grand des péchés en musique $»^{21}$. La formule, dont le style est rehaussé par la forme de la sentence, peut néanmoins, par son tranchant, froisser certains lecteurs, alors même que l'idée en soi, plus amplement développée et assumée comme une opinion personnelle, aurait emporté leur adhésion. Elle est énoncée comme telle dans un article ultérieur, sans que le propos ait perdu sa valeur polémique: « [j]'aime beaucoup mieux, je l'avoue, un chant plein de génie, déparé une fois par une fausse note, que la perfection continue et monotone de la médiocrité qui ne tombe pas parce qu'elle ne s'élève jamais $»^{22}$. Dans un souci d'efficacité, Stendhal varie les tonalités de son propos de façon à dérouter son lecteur dont il contrarie les habitudes de penser tout en conservant son suffrage, renvoyant chacun à ses impressions. Tel est 
le propre du dilettante, de frayer, au nom du plaisir, une voie nouvelle par-delà les chemins autorisés.

D'autres stratégies concourent au même but, développées dans des disciplines connexes. Dans la préface de ses Poésies allemandes publiées en 1830, Nerval s'inscrit lui aussi dans les pas de Mme de Staël, vingt ans après la publication de De l'Allemagne, pour proposer une démarche comparatiste : le jeune auteur, qui avait obtenu une certaine notoriété grâce à la traduction du Faust de Goethe publiée en 1827, se donne pour tâche de poursuivre son propre projet d'exploration du domaine littéraire allemand, se faisant à son tour "passeur » entre deux littératures». Il justifie son approche en ces termes :

Pour moi, j'offre ici des traductions de vif enthousiasme et de premier jet, que je n'ai peut-être pas réussi à faire bonnes, mais qui du moins sont exactes et consciencieuses. Les jugements tout faits n'avancent rien en littérature ; des traductions fidèles peuvent, je crois, davantage ${ }^{23}$.

\section{Régression et révolte, portrait de l'auteur en Prométhée}

Dans l'œuvre de Nerval, la révolte, pour être plus dissimulée, n'en est pourtant que plus profonde : le déploiement de la critique nervalienne témoigne d'une volonté de refonder l'érudition sur les cendres du discours académique. L'approche de leur objet par les deux auteurs diffère significativement : chez Nerval l'érudition sera le prétexte à un déploiement diachronique de l'objet étudié. La démarche nervalienne a pour effet d'occulter l'objet présent en le déployant dans le temps. Le modèle généalogique est alors privilégié. La critique thématique a montré l'importance de la recherche des racines dans l'œuvre de Gérard de Nerval : Serge Zenkine observe ainsi que le Voyage en Orient aussi bien que Sylvie constituent « une régression utopique vers le pays natal $»^{24}$ : la terre maternelle de l'humanité et des religions en Egypte ou celle plus intime du Valois de son enfance. Ce fantasme de l'origine contamine l'ensemble de l'œuvre de Nerval et inclut sa production journalistique : ainsi, dans un article de 1845 à propos des fêtes du Bœuf gras $^{25}$, il refuse de faire la description du cortège du carnaval et se concentre sur un examen de ses origines antiques ${ }^{26}$. L'événement s'efface donc devant la somme érudite qui tâche de rétablir le lien entre le passé et le présent.

Un document exceptionnel, La Généalogie fantastique, rédigé en mars 1841 durant un épisode de crise nerveuse, illustre cette tendance régressive propre à Nerval mais aussi son habitude d'élaborer à partir d'un centre des analogies. Celles-ci sont de natures variées : rapprochements étymologiques, géographiques, onomastiques, linguistiques ou encore héraldiques qui impliquent une érudition souvent pointue. Par exemple, Sylvie Lécuyer (qui a fourni une remarquable transcription de la généalogie ${ }^{27}$ ) observe qu'il analyse son nom de famille "Labrunie » en le rapprochant de l'allemand Bruck (le pont) et de Brunn mais en traduisant par erreur ce dernier mot par «tour » au lieu de "fontaine " (comme dans Schoebrunn qu'il traduit correctement plus loin, car il renvoie à Mortefontaine en Valois). La méthode généalogique a donc vocation à tisser des liens entre des connaissances éparses : ainsi, par ce travail actif de l'érudition, l'auteur met en réseau les identités, ce qui lui permet, au prix de recherches plus ou moins fantasques, de s'associer à la famille des Bonaparte et aux chevaliers d'Othon.

La parfaite intelligibilité de ces correspondances est toutefois aussi inaccessible au profane qu'à l'érudit. Chez Nerval, la notion de savoir est en effet dédoublée : en 
parallèle d'un savoir académique et scientifique, accessible à tous, existe un ensemble de connaissances occultes dont la particularité est d'expliquer le premier et de justifier leur unité. Nerval va donc s'attacher à décrire ce savoir occulte et souterrain et à suivre son émergence épisodique au cours de l'histoire de l'humanité. L'auteur en propose une synthèse dans un chapitre de la nouvelle Cagliostro:

Si l'on s'est bien expliqué les doctrines exposées plus haut, on aura pu comprendre par quelles raisons, à côté de l'Église orthodoxe, il s'est développé sans interruption une école moitié religieuse et moitié philosophique qui, féconde en hérésies sans doute $[\ldots] .^{28}$

Le reste du chapitre décrit les étapes de l'histoire d'un tel savoir à la manière d'une généalogie, évoquant tour à tour le pythagorisme, l'école d'Alexandrie, la Kabbale, les Templiers, la franc-maçonnerie, les Druses, les Rosecroix ou encore l'Italie néoplatonicienne, jusqu'au mysticisme révolutionnaire. Si elle tend à rétablir les maillons d'une chaîne secrète, la critique dilettante, parce qu'elle exhibe des sources alternatives et hétérodoxes, n'en adopte pas moins une posture de révolte. Ainsi, dans un texte intitulé Le Diable rouge consacré à la figure de Satan, Nerval écrit :

On peut lire les détails de cette immense catastrophe dans le Paradis Perdu de Milton, - lequel est fondé tout entier sur l'un des livres dits apocryphes de la Bible qui porte le titre de Livre d'Enoch.

Ce livre a toujours été repoussé des bibles orthodoxes, - parce que l'on avait craint qu'il ne se trouvât dans la rébellion de Satan une certaine grandeur qui séduirait les imaginations humaines ${ }^{29}$.

La violence d'une telle démarche réside moins dans le satanisme supposé de ces ouvrages que dans le geste d'excaver ces sources occultées et de placer leur enseignement au même rang que le savoir institué.

\section{De l'expérience subjective au deuil de l'unité : les apories du discours de savoir}

Cette parole contestataire, si elle ouvre des perspectives nouvelles, n'en est pas moins marquée par la réversibilité propre à tout discours critique. On chercherait en vain chez Stendhal les fondements d'une nouvelle science : dans ses écrits, il enseigne au lecteur le moyen de reconquérir sa liberté, par l'exercice de la pensée critique et l'ouverture à la nouveauté. Nerval quant à lui déplore la ruine de l'édifice des connaissances et tâche d'en reconstruire un nouveau à partir de ses fragments épars.

\section{Le dilettante, un nouveau type de connaisseur}

Dans ses écrits journalistiques, Stendhal exprime des opinions tranchées sans chercher pour autant à les imposer à autrui, considérant que « chacun a raison dans son trou, et qu'il est absurde de vouloir être à la fois dans deux trous $»^{30}$ : c'est davantage pour lui une façon d'appeler la répartie, d'endosser son rôle d' "excitateur d'idées " ${ }^{31}$. Posant comme fondamentale la relativité du goût dont Diderot et d'autres s'étaient prévalus au siècle précédent, il se récrie contre les diktats que les voix autorisées imposent à l'opinion publique à laquelle elles ôtent de la sorte l'occasion de se déterminer. Tel est ce qu'il dénonce à travers cette mise en scène énonciative : 
«Mais, dit-on, le public sifflera tous les opéras nouveaux!» Tant pis pour le public. Mais, jusqu'à ce qu'on ait tenté l'expérience, je me permets de douter du rôle singulier que des voix intéressées lui prêtent par avance ${ }^{32}$. soi-même, Stendhal le rappelle à ses responsabilités : c'est à lui en effet qu'il appartient de décider du succès ou de l'échec d'une représentation. Il tient pour établi que, dans le domaine des beaux-arts, le public est le "souverain juge $»^{33}$, mais estime toutefois qu'il n'est un juge compétent que pour autant qu'il soit «disposé à sentir la musique $»^{34}$. Autre maxime du dilettante : il s'agit avant tout d'éprouver ses sens pour en cultiver les plaisirs. Or juger non en savant, mais "par sentiment $»^{35}$, suppose une faculté d'accueil et d'écoute, d'attention soutenue et d'ouverture à la nouveauté : «[i]l faut [en effet] se prêter un peu à la musique, si l'on veut lui devoir des sensations : elle ne force pas les applaudissements comme les vers ronflants d'une tragédie $»^{36}$. La connaissance, en ce domaine, procède de l'expérience renouvelée par laquelle les sens apprivoisent de nouveaux rapports, qui ont d'abord contre eux la puissance de l'habitude. Si l'appréciation de la musique est affaire de sensations, elle n'en requiert pas moins un perfectionnement du goût. À cet égard, un interprète dispose d'autant mieux le public en faveur des formes nouvelles qu'il produit sur lui une vive impression. Ainsi donc, avec le temps, le public peut apprendre beaucoup d'un grand talent. Et Stendhal de noter avec satisfaction :

[...] le public d'aujourd'hui est sans comparaison supérieur au public d'il y a cinq ans. Il a fait preuve ce soir d'une sûreté de goût et d'une finesse de tact qu'on n'avait jamais remarquées avant le règne de madame Pasta. Cette grande actrice $\mathrm{a}$ initié le public dans tous les mystères de la musique seria ${ }^{37}$.

On relèvera d'emblée la dimension ésotérique du propos : les notions d'initiation et de « mystères » font de la musique une science occulte dont le premier chanteur maîtrise les arcanes, qu'il dévoile progressivement aux initiés, c'est-à-dire, ici, au public des dilettanti. Il importe par ailleurs de noter que les mentions conjointes du " goût » et du "tact», qui semblaient ancrer le propos dans la réalité mondaine des convenances, relèvent bien plutôt de l'appréciation d'un mérite délicat par lequel on atteint à la jouissance esthétique. En effet, le plaisir s'éduque, et c'est même aux yeux de Stendhal le principe qui fonde une appréciation équitable, à laquelle ne saurait prétendre le

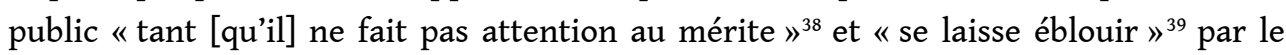
luxe des fioriture sous lequel «les excellents chanteurs $»^{40}$ "étouffe[nt] la pensée du compositeur $"^{41}$. Dans l'esprit de Stendhal, le dilettante, en affinant son goût, concourt au perfectionnement de l'art pour lequel il se passionne. À cette lumière, le public ne saurait être considéré comme un tiers passif : à la fois juge et partie, il participe à la réussite de la représentation en présidant à l'ajustement des diverses interprétations par ses marques de contentement, ou de désapprobation. Rendant compte de l'interprétation de Mlle Mombelli lors d'une représentation de la Dona del Lago, opéra de Rossini, Stendhal regrette que le public n'ait pas sanctionné cette "manière surannée » de chanter en conservant toujours «un air riant $»^{42}$, "quelque [soit] le sentiment de douleur ou de terreur [que puissent] exprimer » les paroles $»^{43}$. C'eût été donner à l'interprète l'occasion de se corriger : «Mlle Mombelli, pour peu qu'elle soit avertie par le silence des spectateurs, a assez de talent pour broder ce duo avec des ornements tristes $»^{44}$. Si donc les interprètes de grand talent sont censés initier le public aux jouissances de l'expérience esthétique, ce dernier en est le garant: sans s'ériger en censeur ${ }^{45}$, il doit veiller au maintien de l'illusion nécessaire au 
développement de ses émotions. En définitive, le dilettantisme selon Stendhal est une économie du plaisir fondée sur la capacité de chacun à sentir par soi-même, «à jouir pour son compte du plaisir et de l'émotion que [la musique] peut lui procurer $»^{46}$. La chronique stendhalienne du Théâtre-Royal italien opère une révolution en faisant procéder le goût d'une absence de connaissance, ou plutôt, d'une connaissance acquise par l'expérience, subordonnant ainsi la science à une approche sensible de l'art. Stendhal en témoigne lui-même dans ses Souvenirs d'Égotisme : « [à] force d'être heureux à la Scala (salle de Milan), j'étais devenu une espèce de connaisseur ${ }^{47}$.

\section{La nostalgie de l'unité}

l'érudition visant à interroger plus largement la position de l'homme au sein de l'édifice des connaissances. Ce pas de côté propre au dilettante grâce auquel Nerval refuse de prendre au part au conflit des légitimités du savoir et tâche de réhabiliter les savoirs réprouvés, le conduit également à adopter un regard réflexif et surplombant, de sorte que son propos s'assimile in fine moins à l'exposé d'un connaisseur qu'à une description des mécanismes de recouvrement, de stratification et d'occultation des connaissances. Poursuivons notre parcours du Voyage en Orient, assimilé ici à une traversée des formes de l'érudition : le voyageur refuse de décrire les monuments de l'ancienne Syrie sous prétexte qu'" on ne peut plus les dépeindre après Volney et Lamartine $\aleph^{48}$. L'examen de l'Orient par Nerval s'assimile donc à un parcours des discours littéraires ou savants qui l'ont façonné. Edward Saïd attribue ainsi à Nerval une place singulière au sein des écrivains orientalistes : il considère que « la pure et simple transcription de textes érudits étaient les seuls vaisseaux possibles " pour cet Orient appartenant « au royaume du négatif ou des récits ratés, des chroniques désordonnées $»^{49}$. En Orient, l'érudition enferme le narrateur dans un cercle herméneutique dont il refuse de s'extraire.

Cette pratique n'est cependant pas inédite à l'Orient ni aux terres antiques. Paul Bénichou observe ainsi que la réhabilitation de la chanson folklorique du Valois par Nerval diffère de celle organisée à la même époque par l'institution scientifique. En 1852, un décret du ministre de l'Instruction publique, Hippolyte Fortoul, organise un projet de sauvegarde des chansons populaires de la France sous l'égide de plusieurs savants (dont J.-J. Ampère, Sainte-Beuve, Nisard et Mérimée). Nerval, loin d'adhérer à une telle entreprise, en dénonce les limites dans un article paru la même année dans L'Artiste :

On publie aujourd'hui les chansons patoises de Bretagne ou d'Aquitaine, mais aucun chant des vieilles provinces où s'est toujours parlée la vraie langue française ne nous sera conservé. [...] Mais songera-t-on à recueillir ces chants de la vieille France dont je cite des fragments épars et qui n'ont jamais été compilés ou réunis. ${ }^{50}$

Paul Bénichou considère ainsi que Nerval propose ici « une critique de l'érudition médiévalisante en tant qu'elle entrave et fausse le contact avec le folklore poétique actuel $\aleph^{51}$. Si Nerval a raison de dénoncer une pratique héritée des philologues qui occulte son objet, Bénichou remarque que les conclusions de l'enquête Fortoul, parues l'année suivante, adopteront la même démarche que Nerval.

Nerval, qui emprunte tout d'abord sa démarche à l'érudit, propose in fine dans ces textes, une pratique singulière qui refuse la somme érudite au profit de la réunion de 
"fragments épars », qu'il convient de rapprocher de celle du dilettante : la critique a bien montré que pour correspondre à cette épistémologie romantique héritée de la tradition allemande, Nerval fera le choix d'un récit excentrique ${ }^{52}$, vif et allègre qui emprunte son paradigme à la promenade plus qu'au voyage ${ }^{53}$.

Le dilettantisme de Nerval est en effet celui d'une érudition qui a fait le deuil de l'unité. Nerval applique ici à l'épistémologie une démarche proche de celle du mysticisme qui consiste à contempler l'unité à travers la fuyante multiplicité des apparences et à travers la réunion des fragments épars de la réalité ${ }^{54}$. En effet, pour Nerval, qui a toujours manifesté une certaine prédilection pour les doctrines mystiques et les pratiques ésotériques, le savoir doit toujours être l'objet d'une initiation, d'un parcours, d'un effort : c'est pour cette raison qu'il refuse dans un même mouvement les savoirs institués et ceux qui tendent à s'élaborer en système fermé. Sa description de la religion druze dans le Voyage en Orient est attentive à ce principe. Largement informée par la somme érudite rédigée par l'orientaliste Sylvestre de Sacy, intitulée Exposé de la religion des Druzes ${ }^{55}$, elle est pourtant présentée de manière singulière : tout d'abord, le récit présente l'entretien du voyageur avec un cheikh druze. Le voyageur, soucieux de se présenter en initié, adopte la posture d'érudit qui ne convainc pas son auditeur. Après avoir évoqué cet échec, le narrateur présente à son lecteur un résumé de ses entretiens, acceptés à contrecœur par le cheikh. Ce texte prend la forme d'une dissertation construite " comme un discours quasi-scientifique ", présenté " d'un ton sec, ce qui ne s'accorde pas très bien à un récit de voyage $»^{56}$.

31 Le premier refus du cheikh, ainsi que la révélation plus tard dans le récit de l'échec du projet de mariage avec sa fille posent le soupçon sur l'adhésion de Nerval à cet exposé qui, à la manière du chapitre de Cagliostro dans les Illuminés évoqué précédemment met en œuvre un syncrétisme vertigineux, convoquant tour à tour Pythagore, la francmaçonnerie, Mahomet, la mythologie grecque, les templiers, la kabbale. Nerval choisit en outre de le compléter dans les appendices du Voyage en Orient d'un «Catéchisme des Druses» présenté sous la forme d'une succession de questions et de réponses ${ }^{57}$ entièrement plagiées sur l'ouvrage de Sylvestre de Sacy, reléguant ainsi la parole de l'érudit mythologue aux marges du texte. En contrepoint de ces deux discours, Nerval propose une dernière synthèse de ses connaissances sur la religion druze à travers un conte, largement influencé par le modèle des Mille et unes Nuits et informé par les sources savantes de Nerval : celui-ci relate l'histoire du calife Hakem, le fondateur de cette spiritualité. Le lecteur de la section "Druses et Maronites" du Voyage en Orient doit donc, à la manière de l'auteur, être attentif aux recouvrements provoqués par le discours érudit et se faire à son tour dilettante en recueillant les fragments de la doctrine druze à travers de multiples sources : le discours de l'érudit, celui du voyageur et celui du poète.

Davantage qu'en érudits dont ils révoquent la posture, Nerval et Stendhal se posent en amateurs savants tout invoquant, à des titres divers, la figure du dilettante. Très critiques à l'égard des savoirs institués, ils se positionnent différemment par rapport à leur objet : Nerval analyse les mécanismes de la production du savoir et leur impensé, déplorant sur le mode élégiaque le recouvrement des connaissances anciennes par les nouvelles, tandis que le propos stendhalien s'inscrit dans une perspective socioculturelle, pointant la dimension institutionnelle de la promotion des discours de savoir hégémoniques et son retentissement sur l'opinion. 

discours : Stendhal prône une révolution du goût dans laquelle la connaissance, issue de l'expérience, s'inscrit dans une économie du plaisir, tandis que Nerval rêve de reconstruire l'édifice des connaissances à partir des fragments d'une unité perdue. Revendiquant tous deux la singularité de leur entreprise, ils rencontrent le même écueil : la réversibilité du discours critique le rend inapte à accueillir les fondements d'une épistémologie nouvelle. Leurs discours témoignent d'une même conscience des apories du discours de savoir, qui fonderait in fine la posture du dilettante.

L'on dira pour finir que l'attitude critique de Nerval et de Stendhal à l'égard des discours de savoir est représentative d'une sensibilité romantique, tendue entre le culte de l'ancien et la fascination du nouveau. Empêtrés dans les contradictions de leur époque, ils préparent l'avènement d'une critique moderne illustrée par Sainte-Beuve et Baudelaire.

\section{NOTES}

1. Serge Zenkine, L'Expérience du relatif. Le romantisme français et l'idée de culture, Paris, Classiques Garnier, coll. «Etudes romantiques et dix-neuviémistes », 2011, p.11.

2. « M. Jay et les pointus littéraires », La Tribune romantique (mai 1830), in CEuvres complètes, t. I, Paris, Gallimard, coll. « Bibliothèque de la Pléiade », 1989, p.900-902.

3. Stendhal doit au succès de sa Vie de Rossini sa reconnaissance comme critique musical et son entrée en tant que tel au Journal de Paris.

4. «La tâche du critique ", article non publié [1822], dans : Paris-Londres. Chroniques, éd. Renée Dénier, Paris, Stock, 1997, p.51.

5. P. Larousse, Grand Dictionnaire universel du XIXe siècle, Paris, Administration du grand Dictionnaire universel, 1866-1877, t.VI, p.852 (article « Dilettante »).

6. Fait remarquable: sous un régime conservateur, on voit les journaux gouvernementaux prendre le parti de la musique italienne, tandis que la presse libérale défend ardemment la tradition française.

7. « Début de Mlle Ferlotti dans La Pastorella feudataria, musique de M. Vaccaï ». Journal de Paris du 23 avril 1827.

8. «Second début de Galli dans La Cenerentola », Journal du Paris du 11 août 1825.

9. Ibid.

10. Ibid.

11. « Deuxième acte de La Clemenza di Tito », art. cit.

12. À en croire Stendhal en effet, «[l]a vanité française est telle qu'un homme de trente ans n'aime pas qu'on lui enseigne quelque chose : un Français est intimement persuadé qu'il sait tout ce qu'il est convenable qu'il sache» [Stendhal, "L'aristocratie parisienne», London Magazine, décembre 1824. Dans Paris-Londres. Chroniques, éd.cit., p. 228].

13. «M. Jay et les pointus littéraires », La Tribune romantique (mai 1830), art. cit.

14. Daniel Lançon, Les Français en Égypte. De l'Orient romantique aux modernités arabes, Presses universitaires de Vincennes, «Littérature Hors Frontière », 2015, p.42.

Belphégor, 17 | 2019 
15. Voyage en Orient, Cuvres complètes, t. II, Paris, Gallimard, coll. "Bibliothèque de la Pléiade ", 1984, p.188.

16. Gérard de Nerval, "Isis», Les Filles du feu, Euvres complètes, t. III, Paris, Gallimard, coll. "Bibliothèque de la Pléiade ", 1993, p.617.

17. Hisashi Mizuno, Nerval. L'écriture du voyage. L'expression de la réalité dans les premières publications du Voyage en Orient et de Lorely. Souvenirs d'Allemagne, Paris, Honoré Champion, coll. « Romantisme et modernités », 2003.

18. Vies de Haydn, de Mozart et de Métastase (1814) : ouvrage publié sous le pseudonyme de L.A.C. Bombet ; Vie de Rossini (1824).

19. «Dernière représentation de Zelmira dans la salle de l'Académie royale de musique ", Journal de Paris du 2 avril 1826.

20. Ibid. Peter von Winter (1754-1825) et Louis Spohr (1784-1859), compositeurs allemands.

21. «Académie royale de musique (par extraordinaire). La première de La Donna del Lago. Début de Mlle Schiassetti » Journal de Paris du 9 septembre 1824.

22. "Début de M. Rubini dans la Cenerentola (6 octobre) ", Journal de Paris du 9 octobre 1825.

23. Poésies allemandes, dans CEuvres complètes, op. cit., t.I, p.263.

24. Serge Zenkine, L'Expérience du relatif. Le romantisme français et l'idée de culture, op. cit., 2011, p.137.

25. « Le Bœuf gras ", L'Artiste, 9 février 1845, op. cit., p.900-902.

26. Michel Brix, Nerval journaliste (1826-1851): Problématique. Méthodes d'attribution, Namur, Presses universitaires de Namur, 1986 p.131.

27. Sylvie Lécuyer, La Généalogie fantastique de Gérard de Nerval : transcription et commentaire du manuscrit autographe, Namur, Presses universitaires de Namur, coll. «Etudes nervaliennes et romantiques ", 2011

28. «Cagliostro », Les Illuminés, CEuvres complètes, t. II, op. cit., p.1122.

29. Le Diable rouge, CEuvres complètes, t. IX, Paris, Classiques Garnier, coll. «Bibliothèque du XIX ${ }^{\mathrm{e}}$ siècle », 2015.

30. Lettre à Adolphe de Mareste, 2 novembre 1819 [Stendhal, Correspondance, éd. H. Martineau et V. Del Litto, Paris, Gallimard, 1968, t.I, p. 994].

31. " M. de Stendhal. Ses Euvres complètes ", Le Moniteur universel, 2 janvier 1854, dans SainteBeuve, Pour la critique, éd. A. Prassoloff et J.-L. Diaz, Paris, Gallimard, 1992, p. 332.

32. "Otello (11 décembre) », Journal de Paris du 15 décembre 1824.

33. " Académie royale de musique (par extraordinaire). La première de La Donna del Lago. Début de Mlle Schiassetti », Journal de Paris du 9 septembre 1824.

34. "Première représentation de la Semiramide. Début de Mlle Mainvielle-Fodor », Le Mercure du XIX $X^{e}$ siècle, 1825, t. XI, p. 470.

35. «Reprise d'Otello. Début de M. Rubini dans le rôle d'Otello ", Journal de Paris du 17 décembre 1825.

36. «Reprise d'Elisa e Claudio, opéra de Mercadante", Journal de Paris du 23 novembre 1826. De fait, d'après Stendhal, «toutes les affectations viennent au secours du tragique, et trouvent leur intérêt à le louer » [« La Cenerentola (27 novembre) », Journal de Paris du 2 décembre 1824].

37. « La Donna del Lago (12 octobre) », Journal de Paris du 15 octobre 1824.

38. « Reprise d'Elisa e Claudio, opéra de Mercadante », Journal de Paris du 23 novembre 1826.

39. "Suite du début de Mlle Pisaroni dans le rôle d'Arsace de Sémiramis ", Journal de Paris du 8 juin 1827.

40. Ibid.

41. Ibid.

42. « Début de Mlle Cesari dans Semiramide », Journal de Paris du 9 octobre 1826.

43. Ibid.

44. « Troisième représentation de La Donna del Lago ", Journal de Paris du 13 septembre 1824. 
45. Une attention pointilleuse, une attitude trop sévère ne réussissent pas mieux : « $[u] n$ public froid nuit à ses plaisirs en paralysant les moyens des chanteurs qui paraissent devant lui ». [ "Début de Mlle Ferlotti dans La Pastorella feudataria, musique de M. Vaccaï », Journal de Paris du 23 avril 1827].

46. « La Donna del Lago (17 mars) », Journal de Paris du 20 mars 1825.

47. Souvenirs d'égotisme, éd. H. Martineau, Paris, Le Divan, 1927, p. 123.

48. Voyage en Orient, op. cit., p.598.

49. Edward D. Saïd, L'Orientalisme. L'Orient créé par l'Occident, Paris, Seuil, coll. "La couleur des idées ", 1980, p.212.

50. "Les Vieilles ballades françaises", L'Artiste, 15 octobre 1852 repris dans La Bohème galante (Euvres complète, t. III, op. cit., p.284).

51. Paul Bénichou, Nerval et la chanson folklorique, Paris, José Corti, 1970, p.331.

52. Voir Daniel Sangsue, Le Récit excentrique, Paris, José Corti, 1987.

53. Voir Philippe Antoine, Quand l'écriture devient promenade, Paris, Presses universitaires de Paris-Sorbonne, coll. «Imago Mundi », 2011.

54. Albert Béguin, Gérard de Nerval, suivi de Poésie et mystique, Paris, Stock, 1936, p.113-114.

55. Antoine Isaac Sylvestre de Sacy, Exposé de la religion des Druzes, 2 volumes, Paris, L'Imprimerie royale, 1838.

56. Hisashi Mizuno, Nerval. L'écriture du voyage, op. cit., p.288.

57. « Catéchisme des Druses », Voyage en Orient, op. cit., p.831-837.

\section{ABSTRACTS}

Empruntant au spécialiste son discours et à l'amateur sa posture, Stendhal et Nerval se situent tous deux en marge des institutions académiques et marquent un jalon de l'histoire culturelle qu'une archéologie des formes de l'érudition des amateurs se doit de prendre en compte. Ces deux auteurs adoptent la posture du dilettante afin de critiquer l'érudition académique à travers ses objets, ses formes et ses médias. Cette révolution romantique renouvelle tant la visée que les destinataires d'un discours critique qui se tient à la charnière du discours académique et du discours de vulgarisation. Cette critique se déploie sans ordre ni méthode, n'ayant pas vocation à s'ériger en système, et se pratique sur le mode de la causerie, la communication ne visant pas nécessairement à instruire mais reposant avant tout sur le plaisir de l'échange. Il s'agit pour eux de renouveler la perception du savoir, considéré non plus du point du vue académique, mais de celui de l'expérience personnelle.

érudition des amateurs, Gérard de Nerval, Stendhal, institution littéraire, légitimité

\section{AUTHORS}

\section{JULIETTE MASCART}

Juliette Mascart est doctorante à la Faculté des Lettres de Sorbonne Université. Elle travaille actuellement sous la direction de Mariane Bury à une thèse intitulée "Revendiquer le statut d'homme de lettres sous la Restauration à travers l'activité journaliste : l'exemple d'Henri Beyle". Dans ce projet de recherche, l'analyse littéraire ouvre sur des questionnements tant 
sociologiques que médiologiques afin de mettre en lien pratiques d'écriture, supports journalistiques et formes de sociabilité héritées de l'âge classique.

\section{RÉMY ARCEMISBÉHÈRE}

Rémy Arcemisbéhère est doctorant à la Faculté des Lettres de Sorbonne Université ; il réalise actuellement une thèse sur le travail des sources dans l'œuvre du poète Gérard de Nerval sous la direction de Sophie Basch. Il est affilié au Cellf. Ses domaines de spécialisation sont le Romantisme français et européen, l'orientalisme, l'étude des sources (génétique, intertextualité et philologie). Il participe au projet "Bibliothèques d'Orient" coordonné par la Bibliothèque nationale de France. 